\title{
ENDERLEINELLUS tAMIASIS FAHRENHOLZ, 1916 (ANOPLURA: ENDERLEINELIDAE), ESPÈCE IMPORTÉE, IMPLANTÉE ET NOUVELLE POUR LA FAUNE DE FrANCE
}

\author{
BEAUCOURNU J.-C.****, PISANU B.**** \& CHAPUIS J.-L.****
}

Summary: ENDERLEINELLUS TAMIASIS FAHRENHOLZ, 1916 (ANOPLURA: ENDERLEINELLIDAE), AN INTRODUCED SPECIES, AND A NEW SUCKING LOUSE FOR THE French FAuna

A new sucking louse is recorded for the French Anopluran fauna, Enderleinellus tamiasis found on the introduced Sciurid Tamias sibiricus. This observation highlights the maintenance of parasites when introduced with their hosts and when their hosts settle into a novel environments. It suggests a common origin for two out of four populations of Siberian chipmunks examined. The authors describe the morphological criteria that allow the distinction between the two species of Enderleinellus and each infecting a sciurid host found in our country.

KEY WORDS : Enderleinellus tamiasis, sucking louse, Tamias sibiricus, France.

\begin{abstract}
Résumé :
Un pou nouveau pour la France, Enderleinellus tamiasis, a été trouvé sur le Sciuridé Tamias sibiricus introduit. Cette observation révèle le maintien de parasites lors de l'introduction et de l'installation de leur hôte dans un nouveau milieu et suggère une origine commune pour deux des quatre populations de tamias examinées. Les auteurs donnent les critères morphologiques permettant de séparer les deux espèces d'Enderleinellus chacune inféodée à l'un des deux Sciuridés porteurs de poux rencontrés dans notre pays.
\end{abstract}

MOTS CLÉS : Enderleinellus, pou, Tamias sibiricus, France.

de Haute-Provence, de Neohaematopinus sciuri Jancke, 1932, pou de l'Écureuil roux (Sciurus vulgaris). Cette capture, effectuée pratiquement à la frontière italienne (quelques kilomètres avant le Col de Larche) doit faire admettre sa présence dans le Piémont, au moins. Il est connu de Suisse (Beaucournu, 1968), mais non d'Italie. Depuis les années 1960, un nouvel animal de compagnie est apparu dans les animaleries françaises: Tamias sibiricus, communément appelé Tamia de Sibérie ou Écureuil de Corée ou Écureuil du Japon. Pour M. Kawamichi, spécialiste de ce sciuridé (com. pers. à J.-L. C.), les premiers animaux vendus en France, originaires de Corée, transitaient par le Japon d'où ils étaient acheminés en Europe. Le Tamia est présent au Japon à l'état sauvage, où il y est protégé. C'est une sous-espèce distincte de celle du continent ( $T$. s. lineatus, $c f$. Ognev, 1940) et peut-être même une espèce différente de T. sibiricus (M. Kawamichi, travaux en cours). Actuellement, les tamias vendus dans les animaleries seraient issus d'élevages présents en France et aux Pays-Bas (J. Loiselay, com. pers. à J.-L. C.).

En 1969 déjà, les promeneurs pouvaient les observer en liberté dans le Jardin des Plantes de Paris entre les rues Buffon et Cuvier, où environ 400 animaux en provenance de Corée avaient été accidentellement introduits (F. Petter, com. pers. à J.-L. C). Cette population s'est maintenue au cours des années suivantes, puis a décliné, pour disparaître en 1977. Par la suite, d'autres populations se sont installées dans des forêts et parcs périurbains d'Ile-de-France à partir d'animaux lâchés 
dans la nature par des particuliers lassés de cet animal ou encombrés par sa progéniture. Au total, une dizaine de populations, les plus souvent florissantes, sont signalées par Chapuis (2005), et ceci essentiellement dans la grande couronne parisienne.

En théorie, les animaux en vente doivent répondre à divers critères, dont leur état sanitaire. Les ectoparasites sont normalement détruits ou disparaissent, soit spontanément, soit du fait des traitements insecticides lors de la phase de captivité. Il peut cependant y avoir des importations non ou peu contrôlées. Le Lapin américain Sylvilagus floridanus, introduit par des chasseurs en manque de gibiers, a conservé sa puce d'origine Euhoplopsyllus glacialis affinis Ewing, 1940, sur la souche élevée dans le Piémont (Beaucournu \& Launay, 1977). C'est également le cas pour l'écureuil gris, Sciurus carolinensis, introduit avec ses poux au moins dans les Iles Britanniques, Enderleinellus longiceps Kellogg et Ferris, 1915, Hoplopleura sciuricola Ferris, 1921 et Neohaematopinus sciuri (cf. Kloet \& Hincks, 1964), (pour ce dernier, bien qu'il soit holarctique, on ne peut éliminer la possibilité d'une contamination aux dépens de Sciurus vulgaris), en même temps, d'ailleurs, qu'une puce spécifique, Orchopeas howardi (cf. Smit, 1957). Pour le Tamia, si aucune puce n'a été introduite avec son hôte (Pisanu et al., 2008), deux stations, sans doute apparentées par leur source d'introduction, nous ont livré son pou, Enderleinellus tamiasis Fahrenholz, 1916. Deux autres sites riches en tamias, le Parc HenriSellier au Plessis-Robinson (Hauts-de-Seine) et surtout la Forêt de Sénart (Essonne) où la population est régulièrement étudiée depuis 2004, n'ont jamais livré cet ectoparasite (Pisanu et al., com. pers.). Nous avons noté également, dans les stations "à poux", un acarien pilicole, spécifique, importé avec son hôte, appartenant à la famille des Listrophoridae.

Le genre Enderleinellus Fahrenholz, 1912, inféodé aux Sciuridés, montre une très vaste répartition (celle de sa famille-hôte) et compte 45 espèces (Durden \& Musser, 1994a). Kim (1966) ne retenait pas le taxon E. tamiasis, mais les dernières révisions de ce genre (et des Anoploures en général), celles de Durden \& Musser (1994a, b) en font état en tant que bonne espèce. Notons que Kim (1966) place le genre Enderleinellus dans les Hoplopleuridae, alors que Kim \& Ludwig (1978), Durden \& Musser (1994 a) en font une famille à part entière, les Enderleinellidae, suivant en cela Kéler (1963). Dans le Catalogue concernant la région paléarctique occidentale évoqué ci-dessus (Beaucournu, 1968), trois espèces du genre Enderleinellus étaient citées :

- E. ferrisi (Touleshkov, 1957), parasite de la Citelle, Citellus (= Spermophilus) citellus, Sciuridé présent, avec son pou, jusqu'en Europe centrale : Bulgarie, Pologne, Macédoine (Durden \& Musser, 1994a);

- E. longiceps Kellog et Ferris, 1915, parasite de Sciurus carolinensis, écureuil néarctique introduit dans les Iles Britanniques;

- E. nitzschi Fahrenholz, 1916, enfin, parasite de Sciurus vulgaris, signalé pour la première fois en France par Séguy (1944), à Richelieu (Indre-et-Loire).

De plus, Beaucournu (1968) citait E. tamiasis, de Russie européenne, signalé par Blagoveshtchensky (1964) : ce pou n'était pas étudié dans le catalogue, sa répartition sortant des limites géographiques de ce travail. Il s'agissait, en fait, d'une espèce nouvelle à l'époque, omise par Beaucournu (op. cit.), E. propinquus Blagoveshtchensky, 1965, parasite de Spermophilus spp, dont S. citellus, pou connu de Russie d'Europe et jusqu'en Syrie et en Turquie. Un autre Enderleinellus avait également été oublié dans la bibliographie de Beaucournu (op. cit.), E. krochinae Blagoveshtchensky, 1965, inféodé à Sciurus anomalus et trouvé, inter alia, en Pologne et en Roumanie (Durden \& Musser, 1994a).

\section{OBJECTIF}

$\mathrm{N}$ otre propos est ici de montrer les différences morphologiques entre les deux espèces du genre présentes en France, E. nitzschi et E. tamiasis. Les Enderleinellus sont tous de très petite taille (adultes, $1 \mathrm{~mm}$ au maximum). Ils sont caractérisés, entre autres, par la présence de deux minuscules plaques sur le sternite abdominal II (Fig. 1 : 5-6). Si l'on considère les poux de Sciuridés français, les deux genres qui leur sont inféodés ne peuvent être confondus, même à l'œil nu, Neohaematopinus mesurant de 1,5 à plus de $2 \mathrm{~mm}$. Par souci d'homogénéité, nous décrirons brièvement E. tamiasis en calquant le texte sur celui qui a été écrit dans le Catalogue (Beaucournu, 1968) à propos de E. nitzschi.

\section{RÉSULTATS}

\section{Enderleinellus tamiasis, Fahrenholz, 1916}

Synonymie : Enderleinellus longiceps Kellog et Ferris, 1915, pro parte auct.; E. propinquus Blagoveshtchensky, 1965 in Blagovestchensky, 1964.

Iconographie : Ferris, 1932; Scanlon, 1955; Blagoveshtchensky, 1964.

Morphologie : on peut noter d'emblée que E. nitzschi et E. tamiasis appartiennent à deux types morphologiquement distincts. E. nitzschi fut décrit en 1818 par Nitzsch, sous le nom pré-occupé par von Olfers (1816), de "sphaerocephalus" : ce nom lui convenait à merveille, la capsule céphalique étant aussi large que longue (Fig. $1: 1$ ). De même, si E. tamiasis a pu être confondu avec E. lon- 

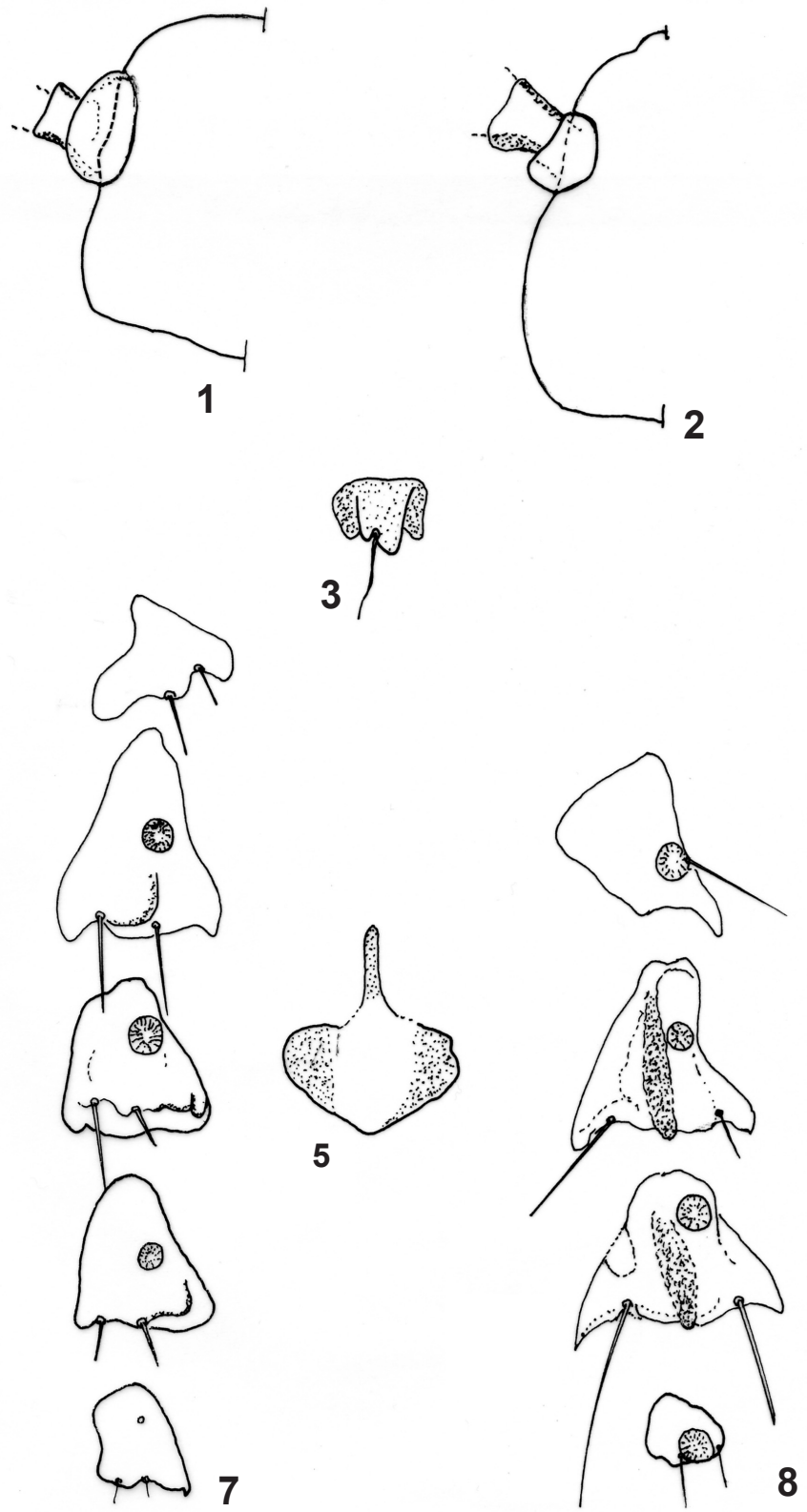

giceps, c'est en raison de la présence, chez les deux espèces, d'une capsule céphalique allongée (Fig. 1 : 2). Il faut toutefois préciser que, à frais, cette différence entre "nitzschi" et "tamiasis" est peu ou non visible, le proboscis incurvé vers la face ventrale chez ce dernier faisant que la capsule céphalique semble tronquée.

Plaque sternale thoracique moins marquée que chez E. nitzschi, le processus médian est peu ou pas visible (Fig. 1 : 3-4). Plaques sternales abdominales aussi larges que longues chez E. nitzschi, beaucoup plus longues que larges chez E. tamiasis (Fig. 1 : 5-6). Les plaques paratergales (Fig. 1 : 7-8) montrent des saillies plus marquées chez E. tamiasis, mais la $4^{\text {ème }}$ plaque est à peine visible et la $5^{\text {ème }}$ obsolète.

Dimensions : E. tamiasis est plus petit que E. nitzschi, pour lequel Beaucournu (1968) donnait les dimensions

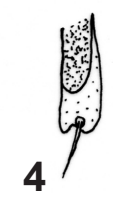

Fig. 1. - Espèces du genre Enderleinellus en France (les chiffres impairs concernent $E$. nitzschi, les chiffres pairs, E. tamiasis). 1-2 : capsules céphaliques en vue dorsale (semi-schématiques); 34 : plaques du sternite II; 5-6 : plaques sternales; 7-8 : plaques paratergales (dessins originaux).

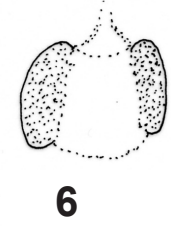

suivantes : 0,86 $\mathrm{mm}$ pour les mâles et 0,96 $\mathrm{mm}$ pour les femelles. Nous avons pu mesurer 10 mâles et 12 femelles de E. tamiasis. La taille moyenne des mâles est de 0, $54 \mathrm{~mm}$ (écarts : 0,51-0,56), celle des femelles est de 0,56 mm (écarts 0,54-0,69).

\section{RÉPARTITION ET MATÉRIEL ÉTUDIÉ}

La répartition a été évoquée plus haut : E. tamiasis est primitivement paléarctique oriental, de la Corée et du Japon, jusqu'en Sibérie, et en Mandchourie. Les populations françaises sont donc, pour le moment, isolées géographiquement.

Pour la France, ce pou est signalé des deux localités suivantes, cette courte liste devant certainement s'enrichir dans les années à venir : 
- Verneuil-sur-Seine (Yvelines), Bois de Verneuil, mai 2006 (1 mâle, 1 femelle conservés in "Ectoparasites Collection at Georgia Southern University", USA, ${ }^{\circ} \mathrm{L}=$ 3388, L. Durden); mai 2007, 4 mâles, 8 femelles, 2 nymphes (dernier stade).

- Sceaux (Hauts-de-Seine), Parc de Sceaux, 13 mai 2007, sur un individu mâle : 7 mâles, 17 femelles, 2 nymphes (dernier stade).

Sur les 19 tamias en provenance du bois de Verneuilsur-Seine, examinés sous loupe binoculaire, des poux ont été trouvés sur trois hôtes. À titre de comparaison, environ la moitié des écureuils roux examinés $(\mathrm{n}=20)$ étaient porteurs d'E. nitzschi, au niveau des zones axillaires. Nous avons collecté ce pou en janvier, décembre et mars. Trois hôtes trouvés écrasés, mais frais, en été, dans le département des Hautes-Alpes, n'étaient pas parasités (un $4^{\mathrm{ème}}$, récolté dans les mêmes conditions, dans les Alpes de Haute-Provence, était porteur de $\mathrm{NeO}-$ haematopinus, $c f$. supra). Le tamia, étant un sciuridé hibernant, les dates de capture ne peuvent se comparer. Les poux semblent se trouver, ici, surtout aux niveaux des épaules et du cou.

\section{REMERCIEMENTS}

Toute notre gratitude va à notre Collègue et Ami le Professeur Lance Durden, Georgia Southern University (USA), qui a bien voulu confirmer notre hypothèse, compléter notre bibliographie et relire ce manuscrit. Nous remercions également la Région Îlede-France, le Conseil général des Hauts-de-Seine et l'Office National des Forêts qui contribuent au financement des travaux réalisés depuis 2004 sur Tamias sibiricus en France.

\section{RÉFÉRENCES}

Beaucournu J.-C. Les Anoploures de Lagomorphes, Rongeurs et Insectivores dans la Région Paléarctique Occidentale et en particulier en France. Annales de Parasitologie humaine et comparée, 1968, 43, 201-271.

Beaucournu J.-C. Présence en France de Neohaematopinus sciuri Jancke, 1932 (Anoplura). Bulletin de la Société entomologique de France, 1990, 94, 309.

Beaucournu J.-C. \& Launay H. Présence en France d'une Xenopsylla selvatique X. cunicularis Smit, 1957 (Siphonaptera, Pulicidae) parasite du lapin de garenne. Bulletin de la Société de Pathologie exotique, 1977, 70, 299-301.

Blagoveshtchensky D.I. (Ordre Anoplura (Siphunculata), in: Clef des Insectes de la partie européenne de l'URSS, Biej-Bienko coord, Académie des Sciences de l'URSS, 1964, 1 (fasc. 17) (en russe).

Blagoveshtchensky D.I. New species of Sucking Lice (Siphunculata) that are parasites of rodents. Communication I. Entomologicheskoe Obozrenie (en russe), trad. angl. in : Entomological Review, 1965, 44, 85-91.
Chapuis J.-L. Répartition en France d'un animal de compagnie naturalisé, le Tamia de Sibérie (Tamias sibiricus). Revue d'Écologie (Terre et Vie), 2005, 60, 239-253.

Davoust B., Boni M., Branquet D., Ducos de Lahitte J. \& MARTET G. Recherche de trois infestations parasitaires chez les rats capturés à Marseille : évaluation du risque zoonosique. Bulletin de l'Académie nationale de Médecine, 1997, 181, 887-897.

Durden L. \& Musser G.G. The Sucking Lice (Insecta, Anoplura) of the World: a taxonomic checklist with records of Mammalian hosts and geographical distribution. Bulletin of the American Museum of Natural History, 1994a, $\mathrm{n}^{\circ} 218,90 \mathrm{p}$.

Durden L. \& Musser G.G. The mammalian Hosts of the Sucking Lice (Anoplura) of the world: a host-parasite List. Bulletin of the Society for Vector Ecology, 1994b, 19, 130-168.

FERRIS G.F. Contributions toward a monograph of the sucking lice. Part V, Stanford University Publications, University Series, Biological Sciences, 1932, 2, 273-413.

GOMEz M.S. Présence à Barcelone de Hoplopleura pacifica (Anoplura, Hoplopleuridae) parasite de Rattus norvegicus. Annales de Parasitologie bumaine et comparée, 1989, 64, 516-517.

Keler S. Ordnung Laüse, Anoplura, in : Brohmer P. et al., Tierwelt Mitteleuropas, 1963, Leipzig, Insekten I, Teil 4, $14 \mathrm{p}$.

KIM K.C. The species of Enderleinellus (Anoplura, Hoplopleuridae) parasitic on the Sciurini and Tamasciurini. The Journal of Parasitology, 1966, 52, 988-1024.

Kim K.C. \& Ludwig H.W. The Family classification of the Anoplura. Systematic Entomology, 1978, 3, 249-284.

KLOET G.S. \& Hincks W.D. A check list of British insects, $2^{\text {nd }}$ ed., Royal entomological Society, London, 1964, 11, part. 1, p. 35.

Marcandier M. \& Pirot R. Étude sur les ectoparasites des rats de Toulon. Bulletin de la Société de Pathologie exotique, 1932, 25, 237-244.

OGNev S.I. Mammals of the USSR and adjacent countries. Vol. IV. Rodents, 429 p., 1940 (en russe), Israel Program for Scientific Translations, Jerusalem, 1966.

Pisanu B., Marmet J., Beaucournu J.-C. \& Chapuis J.-L. Diversité du cortège en Siphonaptères chez le Tamia de Sibérie (Tamias sibiricus Laxmann) introduit en Forêt de Sénart (Ile-de-France). Parasite, 2008, 15, 35-43.

SCANLON J.E. Anoplura from some Korean small mammals hosts. Bulletin of the Brooklyn entomological Society, 1955, $50 \mathrm{p}$.

SÉguY E. Faune de France : Insectes ectoparasites (Mallophages, Anoploures, Aphaniptères). P. Lechevalier éd., Paris, 1944, 43, 684 p.

SMit F.G.A.M. Handbooks for the identification of British Insects. Siphonaptera. Royal entomological Society of London, 1957, 1, part. 16, 94 p.

Voelckel J., Duchassin M. \& Schollhammer G. Variations saisonnières de la faune pulicide de Rattus norvegicus à Marseille. Médecine tropicale, 1964, 24, 2-26.

Reçu le 5 novembre 2007 Accepté le 5 février 2007 